\title{
III. BUCHBESPRECHUNGEN
}

Forstmathematik in Forschung und Unterricht. Von Prof. Dr. S. HopmanN, Vervielfältigt im Rotaprintverfahren bei der Forstlichen Fakultüt Hann.Münden 1951. 235 S. mit 60 Tabellen und 57 Figuren. (Buchhandlung Erwin Mayr, Hann.-Münden, Lange Str. 22.) DM 10,-.

Im Titel und in der Einleitung an das vor über hundert Jahren (1842, nicht 1942, wie zufolge eines Druckfehlers im Lit.-Verz. angegeben) erschienene Lehrbuch von G. KöNIG anknüpfend, behandelt der Verfasser in leicht faßlicher Darstellung, ergänzt durch gut gewählte Beispiele, die Gebiete der angewandten Mathematik, welche für die Forstwissenschaft Bedeutung haben.

Die - man möchte sagen: instinktive - Abneigung gegen die Mathematik, welche in den Kreisen der Forstleute immer noch weit verbreitet ist, wirkt sich als beklagenswerte Hemmung für den Fortschritt auf den meisten forstlichen Sachgebieten aus. Sie hat die unumgängliche Anwendung mathematisch-statistischer Methoden verzögert; mangelnde Kenntnis der Studenten erschwert die Behandlung mancher Stoffgebiete, wie z. B. das der Ertragskunde. Vielen praktischen Forstwirten ist ein verständnisvolles Eindringen in zahlreiche, mit biometrischen Methoden und Begriffen arbeitende Veröffentlichungen verwehrt, weil ihnen die notwendigen Grundlagen fehlen.

So möchte das Buch nicht nur den Studenten und den Forschenden, die hier eine angenehme Zusammenfassung des sonst in vielen. Veröffentlichungen Zerstreuten vorfinden, sondern auch den praktischen Forstleuten zu nachholendem. Studium empfohlen werden.

A s s man $n$.

Messung der Waldbestände. Von Dr. M. PRODAN, Dozent an der Universität Freiburg i. Br. J. D. Sauerländers Verlag, Frankfurt a. M. 1951, 260 S., 71 Übers. und 50 Abb. Ganzleinen DM 16,80.

Die 24 Jahre, welche seit dem Erscheinen von Tischendorfs "Lehrbuch der Holzmassenermittlung" vergangen sind, brachten entscheidende Fortschritte auf dem Wege der „Holzmeßkunde“ alten Stils zu einer "Forstlichen Biometrie“, wie ich das erstrebenswerte Ziel nennen möchte. TischenDORF verdanken wir die erste durchgehende Anwendung der Gaußschen Fehlerrechnung auf die gebräuchlichen Meß3methodiken und Rechenweisen der Holzmeßlehre. HohENADELs führende Gedankengänge, vor allem von dem leider so früh verstorbenen KRENN in ihrer Bedeutung erkannt, ausgebaut und fruchtbar angewendet, zielen darauf $a b$, die verwirrende Vielfalt der Wuchserscheinungen auf wenige, relativ einfache Gesetzmäßigkeiten zurückzuführen und die Bestandskollektive durch Erfassen ho molog e r qua titativer Merkmalwerte klar und gültig zu beschreiben.

Es ist dem Verfasser gelungen, die seit 1927 erschienenen zahlreichen und umfangreichen Arbeiten, insbesondere auch aus den nordischen Ländern und den USA, zusammenfassend auszuwerten und mit dem alten Stoff zu einem organischen Ganzen zu gestalten. Das Buch schließt sich mit der Stoffeinteilung ziemlich eng an die bislang übliche der holzmeßk undlichen Lehrbücher von TISCHENDORF und U. MüLlER an, was hinsichtlich des Anschlusses an die Vergangenheit und der Bedürfnisse der forstlichen Praxis wohl einen Vorteil bedeutet. Die gebrachten Ableitungen sind bei hinreichender mathematischer Strenge klar und gut verständlich, wofür dem Verfasser im Minblick auf die geringen Sympathien, welche der Durchschnittsforstmann mathematischen Dingen entgegenzubringen pflegt, besondere Anerkennung gebührt. Zahlreiche, gut gewählte Beispiele erleichtern das Verständnis und die praktische Anwendung des Gebotenen.

Alles in allem: Ein ausgezeichnetes Werk, das nicht nur den Studierenden, sondern auch den praktisch tätigen Forstwirten uneingeschränkt empfohlen werden kann.

$$
\text { Assmann. }
$$

Die Deutsche Zentralstelle für forstliche Bibliographic hat im Juli 1951 den Jahrgang 1950 der deutschen forstlichen Bibliographie herausgebracht. Eine beschränkte Anzahl von Exemplaren kann zum Preise von DM 6,- pro Stïck von der Deutschen Zentralstelle für forstliche Bibliographie, Freiburg i. Br., Fürstenbergstr. 21, bezogen werden.

Der in Heft 8 erschienene Aufsatz von Oberforstrat Dr. RodeNwaldT ist eine stark gekürzte Bearbeitung einer unter Leitung von Dr. habil. R. B. HILF an der Universität Freiburg eingereichten Dissertation. 